\title{
A vibrational energy harvester based on Soft-Nonlinearity for truly random excitation
}

\author{
Carlo Trigona ${ }^{1,2}$, Jaakko Palosaari ${ }^{1}$, Yang Bai ${ }^{1}$ \\ ${ }^{1}$ Microelectronics Research Unit, University of Oulu, \\ 90014 Oulu, Finland \\ ${ }^{2}$ D.I.E.E.I., Dipartimento di Ingegneria Elettrica Elettronica e \\ Informatica, University of Catania, Viale Andrea Doria 6, \\ 95125, Catania, Italy
}

\begin{abstract}
In this paper, we present a nonlinear energy harvester that is based on a "soft-mode" nonlinearity and is able to work in presence of a truly random excitations. The proposed harvester is configured with a cantilever beam structure, and, at the tip is a cylindrical container filled with freely moving iron balls. The nonlinearity is implemented through the container, as a piecewise function. This structure, in presence of noise, can be assumed as a second order (mass-spring-damper) nonlinear system where the length of the spring changes as a function of external vibration. As will be demonstrated, this nonlinearity will improve the performance of the energy harvester under random excitation. In comparison, the conventional approach based on resonant oscillators is able to collect energy only around its mechanical natural frequency, while the solution pursued here will present a wide spectrum of response. Furthermore, the implemented nonlinearity here does not possess any barrier of potential or mechanical threshold. Because of this, it is able to work at weak signal levels and without mixture of periodic signals. A piezoelectric element has been used to convert the mechanical vibrations into an electrical signal. The system has been modeled and simulated. Experimental validations have been carried out, demonstrating the suitability of the proposed solution.
\end{abstract}

Keywords-soft nonlinearity, nonlinear transducer, energy harvesting, truly random excitation

\section{INTRODUCTION}

Kinetic energy harvesters relying on inertial movement of an equivalent spring-mass dynamic system, no matter with piezoelectric, electromagnetic or electrostatic effects as the energy conversion mechanism, are well known for their resonance phenomena thus narrow bandwidth $[1,2]$. These harvesters only show their optimized performance at their resonant frequencies, and usually lose over $90 \%$ of the optimized performance if the input excitation frequency is shifted over $5 \%$ away from the resonant frequency [2-4]. Introducing nonlinearity is a proven method to significantly broaden the bandwidth. For instance, previous research [3-6] have fabricated arrays of individual harvesters. Each harvester corresponded to certain resonant frequency so the multiple harvesters in an array could cover a broadened frequency range compared to an individual harvester. Magnetic coupling [7,8] or differently oriented laminations [9] have been added to conventional cantilever-structured piezoelectric energy harvesters in order to create multiple potential wells (i.e. more than one stabilized status), so that multiple resonant frequencies thus broadened frequency range could be achieved with the same harvester. In addition, complex beam shapes [10-13], asymmetric tip mass distributions [3], plucking effects [14,15] etc., are also common methods to obtain wideband kinetic energy harvesters. It should be noted that most nonlinear configurations either increase the structural complexity and thus size of a harvester or compromise its maximum output compared to linear counterparts [16]. This may not be favoured in practice when space is limited for the harvester or the output power is also a crucial feature. Researchers attempted to tackle this issue by introducing a fluidic tip mass [17] or a T-shaped beam [13]. The fluidic (honey-motor oil mixture encased in a sealed box) tip mass helped to broaden the bandwidth from $4 \mathrm{~Hz}$ to $10 \mathrm{~Hz}$ without degrading the harvested power [17]. Similarly, the T-shaped beam was able to increase the bandwidth by over $200 \%$ without affecting the peak output power [13].

Nevertheless, although the bandwidth of kinetic energy harvesters can be significantly broadened by introducing nonlinearity, there is still a definite working frequency range beyond which the harvester will still fail to deliver a decent amount of output power. This is because in any nonlinear configuration mentioned above, a single- or multi-well potential with a clear boundary/barrier always exists. The steep boundary defines the edges of the working frequency range. Here, we call this a 'hard' nonlinearity. It is capable of broadening the bandwidth towards certain limit, however, it is not suitable for truly random excitations (e.g. Gaussian noise). In fact, this family of nonlinearity is strictly correlated with the amplitude of the vibrations and with its barrier of potential. For this reason, very often, if the magnitude of external noise is not enough to exceed the barrier a mix between noise and periodic signal is required [18].

In this paper, we introduce a 'soft' nonlinearity in which a steep-boundary well in the potential (as is in the 'hard' nonlinearity case) cannot be identified. As a consequence, the system is able to work in presence of truly random excitation and weak level of signals also thanks to the absence of mechanical thresholds and barriers. In fact, it enables the configuration to constantly and autonomously tune its response, in order to adapt with the random input vibration. Both the simulations and experimental validation have been carried out, point towards a new option for harvesting ambient noise. The paper is organized as follows: 
Section II will describe the working principle, modeling and simulations. Section III reports the experimental setup and the results, while the concluding remarks are given in Section IV.

\section{WORKING PRINCIPLE AND SIMULATIONS}

The energy harvester is based on a cantilever beam in which one end of the structure is fixed whereas the other end moves freely. At the tip a cylindrical container is fixed with the balls inside in order to implement the "soft" nonlinearity, which is very similar to a piecewise function. It is worth noting that, this nonlinearity will improve the performance of the energy harvester in presence of truly random excitation. In fact, as respect the classical approach based on resonant oscillators able to collect energy only around its mechanical natural frequency, the solution pursued here will present a wide spectrum of response, thanks to the "soft" nonlinear behavior. This effect will collect more energy considering that in the vast majority of cases, ambient vibrations assume different forms and the energy is distributed over a wide spectrum of frequencies [18]. Examples of environmental sources are: natural noise signals, induced oscillations, vehicle motions, multi-tone vibrating systems and noisy environments.

Under the hypothesis that the system is excited with wideband noise, non-distributed mass, movement of the mass only along $\mathrm{x}$ axis, vibrations imposed along $\mathrm{z}$ axis, the system can be assumed as a stochastic nonlinear second order system (mass-spring-damper stochastic equation) as expressed in equation 1 :

$$
m \ddot{x}+d \dot{x}+\frac{\partial U(x, \sigma)}{\partial x}=\left.\sigma(t)\right|_{U(x, \sigma)=\frac{E w t^{3} 1}{L(\sigma)^{3} 2} x^{2}}
$$

Where $m$ and $d$ are the mass and the damping coefficient respectively. The term $x$ is the tip displacement of the beam and the dotted terms represent the first and the second derivate of the displacement (velocity and acceleration of the cantilever, respectively). $U(x, \sigma)$ is the potential energy function. It is a function of the displacement and of the noise source $(\sigma)$, and it is a parabola with an elastic constant correlated with the young modulus of the beam $(E)$, the width $(w)$, the thickness $(t)$ and the length of the beam $(L)$. It should be noted that the latter parameter is a variable as a function of the level of vibration.

In presence of noise the potential energy function will change its shape as a consequence of the "random" variation of the term $L(\sigma)^{3}$. The final response is a piecewise function which will be shown through simulations.

Fig.1 shows the schematic of the investigated system and Table 1 lists the parameters of the conceived device.

In order to simulate the proposed energy harvester from random vibrations, the analytical model (see eq.1) has been simulated by using MATLAB ${ }^{\circledR}$ Simulink. Fig. 2 shows the displacement of the oscillator in presence of noise having a wideband distribution from DC to $100 \mathrm{~Hz}$. This is the typical frequency range of the ambient environment $[18,19]$.

A maximum variation of about $1 \mathrm{~cm}$ has been obtained in presence of a RMS force of 0.0174 N. Fig. 3a shows the effect of the variation of the term $L(\sigma)$ in the potential energy function. As can be observed, various parabolas can be assumed as a function of the displacement. Fig. $3 \mathrm{~b}$ shows the close up in the range of $3.1-4.5 \times 10^{-3} \mathrm{~m}$. It evinces the jumping from one potential energy function to another one as a consequence of the noisy vibration.

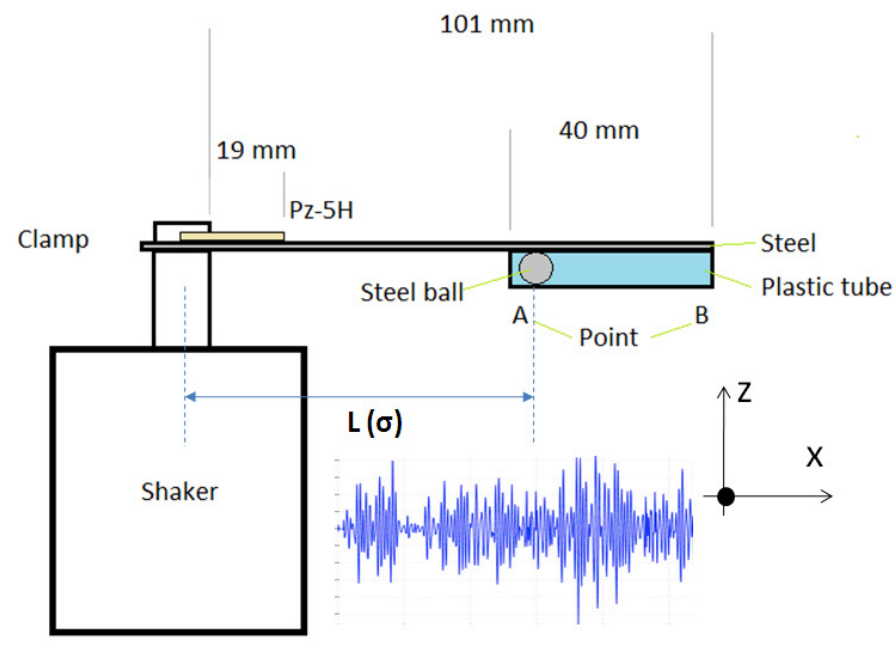

Fig. 1. Nonlinear energy harvester based on "soft" nonlinearity and able to work in presence of truly random excitation.

TABLE I. PARAMETER OF THE CONCEIVED DEVICE

\begin{tabular}{|c|c|c|}
\hline Parameter & Value & $\begin{array}{c}\text { Unit of } \\
\text { measurement }\end{array}$ \\
\hline $\mathrm{m}(1 \mathrm{ball})$ & $4 \times 10^{-3}$ & $\mathrm{~kg}$ \\
\hline $\mathrm{d}$ & 0.0044 & $\mathrm{~kg} / \mathrm{s}$ \\
\hline $\mathrm{E}$ & 190 & $\mathrm{GPa}$ \\
\hline $\mathrm{w}$ & $10 \times 10^{-3}$ & $\mathrm{~m}$ \\
\hline $\mathrm{t}$ & $250 \times 10^{-6}$ & $\mathrm{~m}$ \\
\hline $\begin{array}{c}\text { Lithout balls }) \\
\text { Ball diameter }\end{array}$ & $101 \times 10^{-3}$ & $\mathrm{~m}$ \\
\hline $\begin{array}{c}\text { Number of } \\
\text { balls }\end{array}$ & Variable $1-4$ & - \\
\hline $\begin{array}{c}\text { Plastic tube } \\
\text { diameter }\end{array}$ & $12.5 \times 10^{-3}$ & $\mathrm{~m}$ \\
\hline $\begin{array}{c}\text { Piezoelectric } \\
\text { material } \\
\text { (PZ-5A) }\end{array}$ & $0.5 \times 39 \times 10$ & $\mathrm{~mm}^{3}$ \\
\hline
\end{tabular}




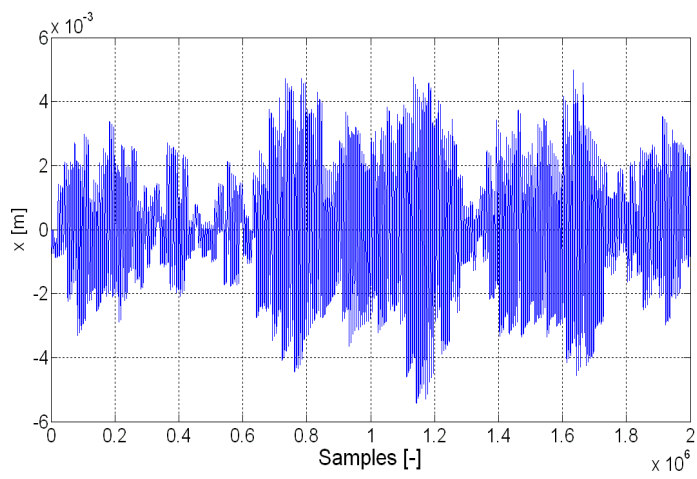

Fig. 2. Simulation: displacement of the tip of the beam in presence of a wideband (from DC to $100 \mathrm{~Hz}$ ) random excitation.

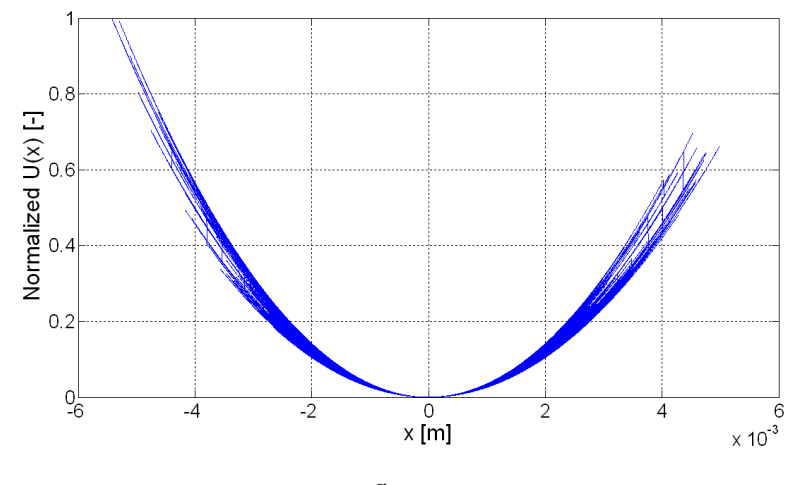

$a$

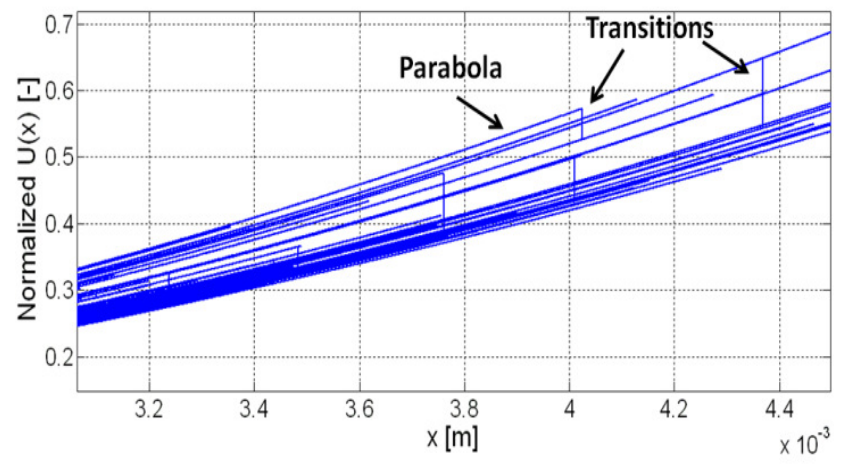

$b$

Fig. 3. Simulations: a) normalized potential energy, b) zoom in order to show the nonlinear effect in terms of transitions between several parabolas. The normalization has been accomplished as respect the maximum value of the potential.

\section{SETUP AND RESULTS}

The components of the cantilever-type energy harvester were machined with laser (ProtoLaser U3, LPKF Laser \& Electronics AG, Germany), providing precise dimensions. A piezoelectric (PZ-5A) patch was machined to $10 \mathrm{~mm}$ wide, $40 \mathrm{~mm}(39.0 \mathrm{~mm}$ for the unclampled part) long and $500 \mu \mathrm{m}$ thick. The patch was covered with $10-15 \mu \mathrm{m}$ nickel electrodes on both sides. A piece of steel as the passive layer was machined to $10 \mathrm{~mm}$ wide and $120 \mathrm{~mm}(101 \mathrm{~mm}$ for the unclampled part) long. The thickness corresponds to $250 \mu \mathrm{m}$. The piezoelectric patch was attached on the steel with a conductive epoxy. A plastic tube containing steel balls (weighed approximately $4 \mathrm{~g}$ for each) moving freely inside was securely attached beneath the free moving cantilever with an insulating tape. The diameter of the steel balls were $10 \mathrm{~mm}$ and the inner volume of the plastic tube as the freely moving space was $52 \mathrm{~mm}$ in length and $12.5 \mathrm{~mm}$ in diameter. A counterweight was added to the opposite end of the cantilever in order to balance forces experienced by the shaker thus to improve dynamics of the shaker.

In order to study the system and its dynamics, a suitable experimental setup was conceived, as shown in Fig. 4. It consists of a signal generator and an amplifier to drive an electro-dynamical shaker (Bruel\&Kjaer) thus to induce the mechanical vibrations. A vibrometer (OFV-5000, Polytec $\mathrm{GmbH}$, Germany) was used to measure the movement of the shaker. The nonlinear energy harvester (the beam with the characteristics presented in Table1) was fixed at the anchor in order to convert the vibration into electric signals. An oscilloscope was used to acquire the output signal of the piezoelectric element.

The system was characterized in terms of number of balls inserted in the cylindrical container. In addition, a comparison between linear and nonlinear behavior was also accomplished by blocking the balls in two known positions, A and B respectively (see Fig. 1).

Fig. 5 shows the normalized PZT output voltage (respect to the maximum output voltage) as a function of the number of balls. The system was excited by a random excitation with the bandwidth of DC to $\sim 100 \mathrm{~Hz}$. The harvested electric signals were extracted from a $250 \mathrm{k} \Omega$ resistive load connected with the harvester, in order to calculate the output power. $50 \mathrm{~s}$ of the output signals were collected. It can be seen (in Fig. 5) that the decrease of the number of balls increased the output voltage obtained across the PZT element. This motivates the consideration that with the increase of the number of balls, the dynamics of the system differed from the model (eq.1) and thus the hypotheses were not fully respected (i.e. non-distributed mass).

Furthermore, the system was also compared with the linear regime, as shown in Fig. 6. It included the piezoelectric output voltage using various balls. The result confirms that the nonlinearity was accentuated in the presence of a single ball and that the nonlinear system presented higher output voltage compared to that of the liner case.

Fig. 7 shows the PZT output waveform with 1 ball and 4 balls as the proof mass. It can be seen that a decrement in terms of amplitude was clearly visible when the number of balls increased. A maximum output voltage of about $30 \mathrm{~V}$ was obtained across the resistive load. 


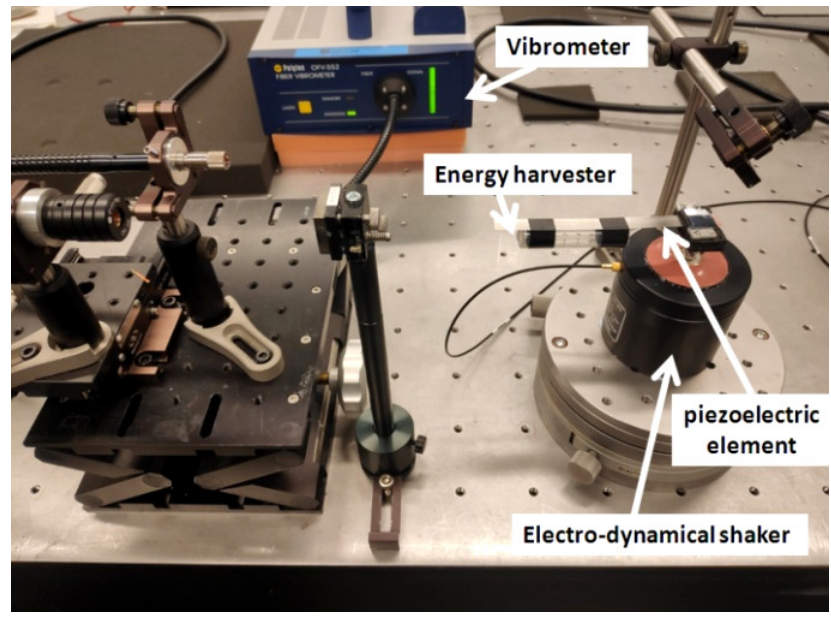

Fig. 4. Experimental setup.

The analysis was also conducted in terms of Power Spectral Density (PSD), in particular a wider spectrum appeared when a single ball is used, see Fig. 8. The result evinces that the 'soft' nonlinearity considered here, which is based on the absence of mechanical threshold and energy barriers (typically presented in nonlinear energy harvesters such as multistable devices [18]), is able to improve the performance of energy harvesters under vibration of truly random excitation and weak signals levels.

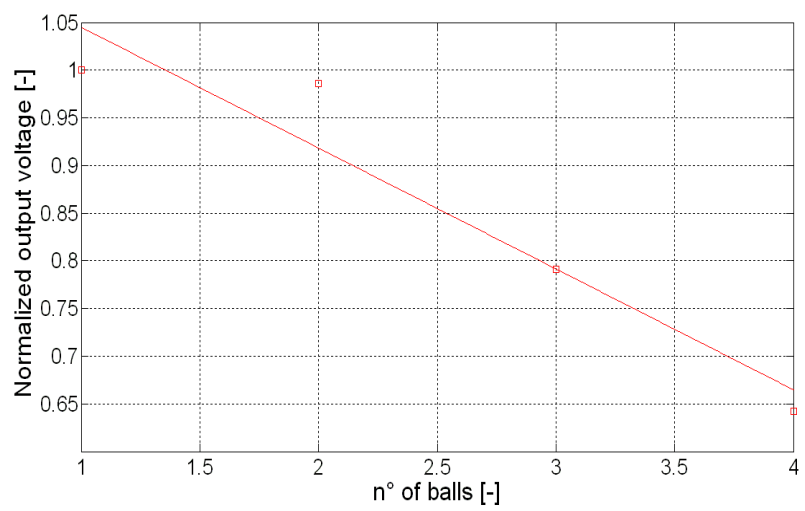

Fig. 5. Normalized PZT output voltage as a function of the number of balls.

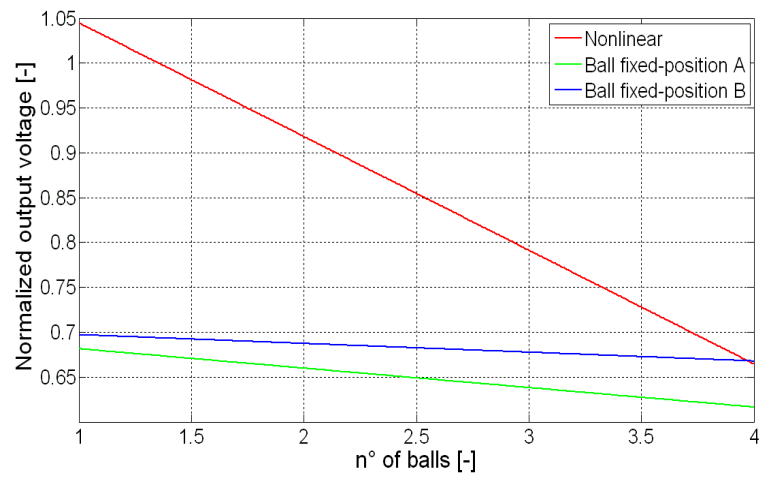

Fig. 6. Normalized PZT output voltage as a function of the number of balls. The graph includes the comparison between the nonlinear system and the lineal condition (ball(s) fixed in the position A or B, as shown in Fig. 1).

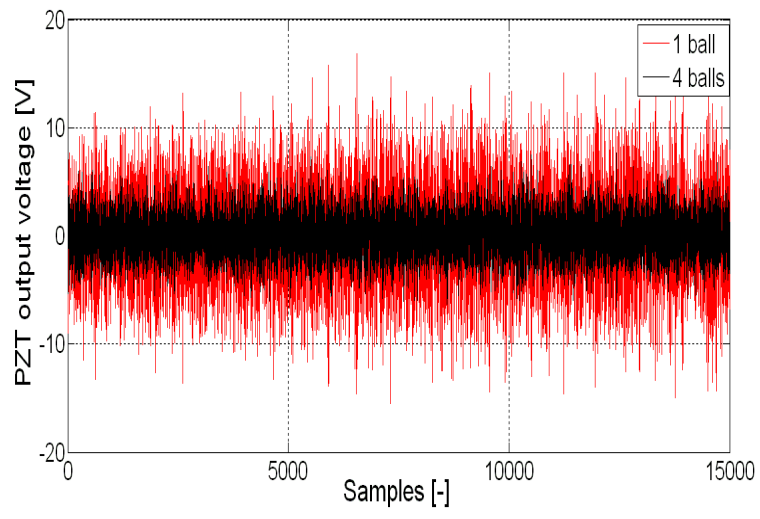

Fig. 7. PZT output voltage across the load resistor $(250 \mathrm{k} \Omega)$ in presence of 1 ball and 4 balls as proof mass respectively.

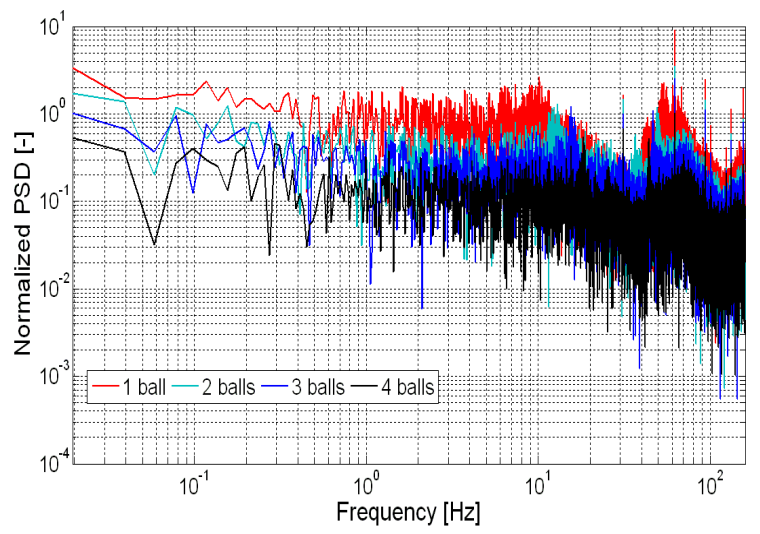

Fig. 8. Normalized PSD for several numbers of balls.

\section{CONCLUSIONS}

In this paper, the possibility of using a soft-nonlinearity to improve the performance of vibrational energy harvesters in presence of truly random excitation has been presented. The system is composed of a nonlinear oscillator with a cylindrical container at its tip filled with balls, in order to implement a piecewise function. The system has been excited through a wideband noise. This nonlinearity does not present any barrier of potential or mechanical threshold, so it is very intriguing to be used in energy harvester able to work under random excitation without the mixture of periodic signals and at weak signals levels. Through the proposed configuration, the response of the system is wider in the spectrum domain, so it is an interesting solution to collect more energy from ambient vibration that typically shows this feature. This points towards a new option for nonlinear harvesting in presence of truly random excitation, which can be used for autonomous or quasi-autonomous sensors and self-sustained measurement systems.

\section{REFERENCES}

[1] Y. Bai, H. Jantunen and J. Juuti, "Energy Harvesting Research: The Road from Single Source to Multisource," Adv Mater, vol. 30, pp. 1707271, AUG 23. 2018.

[2] Y. Bai, P. Tofel, Z. Hadas, J. Smilek, P. Losak, P. Skarvada and R. Macku, "Investigation of a cantilever structured piezoelectric energy harvester used for wearable devices with random vibration input," Mech.Syst.Signal Proc., vol. 106, pp. 303-318, JUN. 2018. 
[3] Y. Bai, C. Meggs and T.W. Button, "Investigation of Using FreeStanding Thick-Film Piezoelectric Energy Harvesters to Develop Wideband Devices," Int.J.Struct.Stab.Dyn., vol. 14, pp. 1440016, DEC. 2014.

[4] Y. Bai, Z. Havranek, P. Tofel, C. Meggs, H. Hughes and T.W. Button, "Nonlinear piezoelectric devices for broadband air-flow energy harvesting," Eur.Phys.J.-Spec.Top., vol. 224, pp. 2675-2685, NOV. 2015.

[5] J. Liu, H. Fang, Z. Xu, X. Mao, X. Shen, D. Chen, H. Liao and B. Cai, "A MEMS-based piezoelectric power generator array for vibration energy harvesting," Microelectron.J., vol. 39, pp. 802-806, MAY. 2008.

[6] Z. Xiao, T.Q. Yang, Y. Dong and X.C. Wang, "Energy harvester array using piezoelectric circular diaphragm for broadband vibration," Appl.Phys.Lett., vol. 104, pp. 223904, JUN 2. 2014.

[7] J. Lin, B. Lee and B. Alphenaar, "The magnetic coupling of a piezoelectric cantilever for enhanced energy harvesting efficiency," Smart Mater.Struct., vol. 19, pp. 045012, APR. 2010.

[8] L. Tang and Y. Yang, "A nonlinear piezoelectric energy harvester with magnetic oscillator," Appl.Phys.Lett., vol. 101, pp. 094102, AUG 27. 2012

[9] D.N. Betts, H.A. Kim, C.R. Bowen and D.J. Inman, "Optimal configurations of bistable piezo-composites for energy harvesting," Appl.Phys.Lett., vol. 100, pp. 114104, MAR 12. 2012.

[10] H. Wu, L. Tang, Y. Yang and C.K. Soh, "Development of a broadband nonlinear two-degree-of-freedom piezoelectric energy harvester," J Intell Mater SystStruct, vol. 25, pp. 1875-1889, SEP. 2014.

[11] I. Izadgoshasb, Y.Y. Lim, L. Tang, R.V. Padilla, Z.S. Tang and M. Sedighi, "Improving efficiency of piezoelectric based energy harvesting from human motions using double pendulum system," Energy Conv.Manag., vol. 184, pp. 559-570, MAR 15. 2019.

[12] C. Lan, L. Tang, W. Qin and L. Xiong, "Magnetically coupled dualbeam energy harvester: Benefit and trade-off," J Intell Mater SystStruct, vol. 29, pp. 1216-1235, APR. 2018.

[13] Z. Xie, T. Wang, C.A.K. Kwuimy, Y. Shao and W. Huang, "Design, analysis and experimental study of a T-shaped piezoelectric energy harvester with internal resonance," Smart Mater.Struct., vol. 28, pp. 085027, AUG. 2019.

[14] Y. Kuang and M. Zhu, "Design study of a mechanically plucked piezoelectric energy harvester using validated finite element modelling," Sens.Actuator A-Phys., vol. 263, pp. 510-520, AUG 15. 2017.

[15] M. Pozzi and M. Zhu, "Plucked piezoelectric bimorphs for knee-joint energy harvesting: modelling and experimental validation," Smart Mater.Struct., vol. 20, pp. 055007, MAY. 2011.

[16] M.S.M. Soliman, E.M. Abdel-Rahman, E.F. El-Saadany and R.R. Mansour, "A wideband vibration-based energy harvester," J MicromechMicroengineering, vol. 18, pp. 115021, NOV. 2008.

[17] M.D. Dhone, P.G. Gawatre and S.S. Balpande, "Frequency band widening technique for cantilever-based vibration energy harvesters through dynamics of fluid motion", Materials Science for Energy Technologies, vol. 1, pp. 84, 2018.

[18] C. Trigona, N. Dumas, L. Latorre, B. Andò, S. Baglio, P. Nouet, "Exploiting benefits of a periodically-forced nonlinear oscillator for energy harvesting from ambient vibrations", Procedia engineering, 25, pp. 819-822, 2011.

[19] N.G. Stephen, "On energy harvesting from ambient vibration", Journal of sound and vibration, 293(1-2), pp. 409-425, 2006. 